\title{
Towards urban crowd visualization
}

\author{
I. Rivalcoba',A, L. Toledo ${ }^{2, A}$, I. Rudomín,B \\ A Barcelona Super Computing Center \\ B Centro de Ciencias de la Complejidad \\ ${ }^{1}$ ORCID: 0000-0002-7070-1625, jrivalco@bsc.es \\ 2 ORCID: oooo-0o02-1196-9189, leonel.toledo@bsc.es \\ 3 ORCID: 00oo-0oo2-1672-1756, rudomin.isaac@gmail.com
}

\begin{abstract}
$\underline{\text { Abstract }}$
Crowd simulation and visualization is an emergent research area that studies and reproduces this phenomenon on virtual environments. We present a system designed for simulation and visualization of pedestrians in urban environments, this system is focused on solving problems such as rendering, character animation, artificial intelligence to deal with steering behaviors and motion planning of each pedestrian. The present work incorporates steering pedestrian behaviors using real data collected from several sources such as GPS traces and surveillance systems, all these data allows virtual characters to adapt their navigation according to several environment factors such as other agents or obstacles. This kind of approach requires massive quantities of resources, such as data, memory and computation. We present a formulation that includes a multi-agent system that assigns individual characteristics, both physical and psychological to the virtual agents, which are based on data obtained from real pedestrians to better depict reality.

The presented system is able to simulate crowds in complex urban environments; for that purpose the system was built in two stages, urban environment generation and pedestrian simulation, for the first stage we integrate the WRLD3D plug-in with real data collected from GPS traces, then we use a hybrid approach done by incorporating steering pedestrian behaviors with the goal of simulating the subtle variations present in real scenarios without needing large amounts of data for those low-level behaviors, such as pedestrian motion affected by other agents and static obstacles nearby.
\end{abstract}

Keywords: crowd simulation, steering behavior, data driven simulation, urban environments.

\section{Introduction}

The process of constructing virtual cities and populate them with virtual agents is increasing as a research area in computer graphics and artificial intelligence. Developing environments with intelligent agents implies several challenges, for instance, rendering thousands of objects within any given scene with geometric and topological variety is complex and many computational resources such as memory and processing power are required. A wide range of areas such as games movies, or urban simulation require virtual $3 \mathrm{D}$ city models with detailed geometry.

Additionally, Agent-based simulation of credible actors in large-scale urban environments is a growing research domain, with numerous applications ranging from security to crisis management, entertainment, urban planning and virtual training [Navarro11]. Simulating hundreds of thousands of individual agents within a very large environment like an airport, a crowded train or station or a whole mega city requires significant computational power. The generation of large urban environments typically involves different stages. Heavily simplifying this model based on a fixed grid layout, a city of finite extent can be generated for a single 
view on the CPU and rendered efficiently on a GPU. Level of detail techniques work properly. Those techniques tend to adapt the complexity of the $3 \mathrm{D}$ models based on the viewpoint of the observer by limiting the number of polygons displayed by the graphic engine in each frame of simulation. Therefore, meshes with a high polygon count often must be simplified to achieve acceptable display rates [Sullivano2].

\section{Related Work}

The creation of urban environments involves a lot of different but related tasks such as the processing of geographic information, the visualization of crowds and the generation of steering behaviors [Silverira2006]. All above with the goal of producing a simulation useful and plausible. In the following paragraphs we discuss some of the relevant works that addresses the tasks enlisted previously.

\section{Geographic Information}

The rising of geographic information systems and data acquisition have led to increase the demand of visualization software in terms of $3 \mathrm{D}$ globe-based interfaces, consequently the need for develop algorithms to reconstruct $3 \mathrm{D}$ data using primarily $2 \mathrm{D}$ objects was increased in the same order. An example of this is the work presented by Essen which describes a method to produce $3 \mathrm{D}$ maps taking as a base $2 \mathrm{D}$ city maps containing relevant features. [Essen2008], by using GPS traces we were able to extract urban and city information to create complex environments using real data and combining them with an interactive crowd, on figure 1 it can be seen a visualization of people's average flow registered by GPS sensors, the greener the street, the higher the stream of people walking on the street.

On the other hand, the work of Thomsen et al. [Thomsen2oo8] introduces a general approach for modeling in 3D GIS addressing the problem of using $3 \mathrm{D}$ data in comparison with traditional $2 \mathrm{D}$ and $2.5 \mathrm{D}$ and how the context of topological abstraction influences the result, depending on the operations to a certain set of data. Using a cell layout, hierarchies are created, and geometry can have a mesh representation.

\section{Crowd Visualization}

The kind of videogames with detailed urban virtual worlds where the players can explore, and approach objectives freely has become a success in the market. The process of creating such environments also known as Open Worlds takes many hours of work. To face this scenario researchers have used procedural modeling via shape grammars with production rules that iteratively evolve a design by increasing the details. The main advantage of this kind of techniques is that the creation of the hierarchical structure is specified in the modeling process. This semantic information allows to reuse design rules bringing forth procedural variations and then creating a large variety of architecture flooding a whole city. However, the completion of this process can take hours and consumes a huge amount of memory (tera-bytes of storage), when rendering a city like Manhattan, which consists of more than 100,00o buildings [Loviscacho6]. 


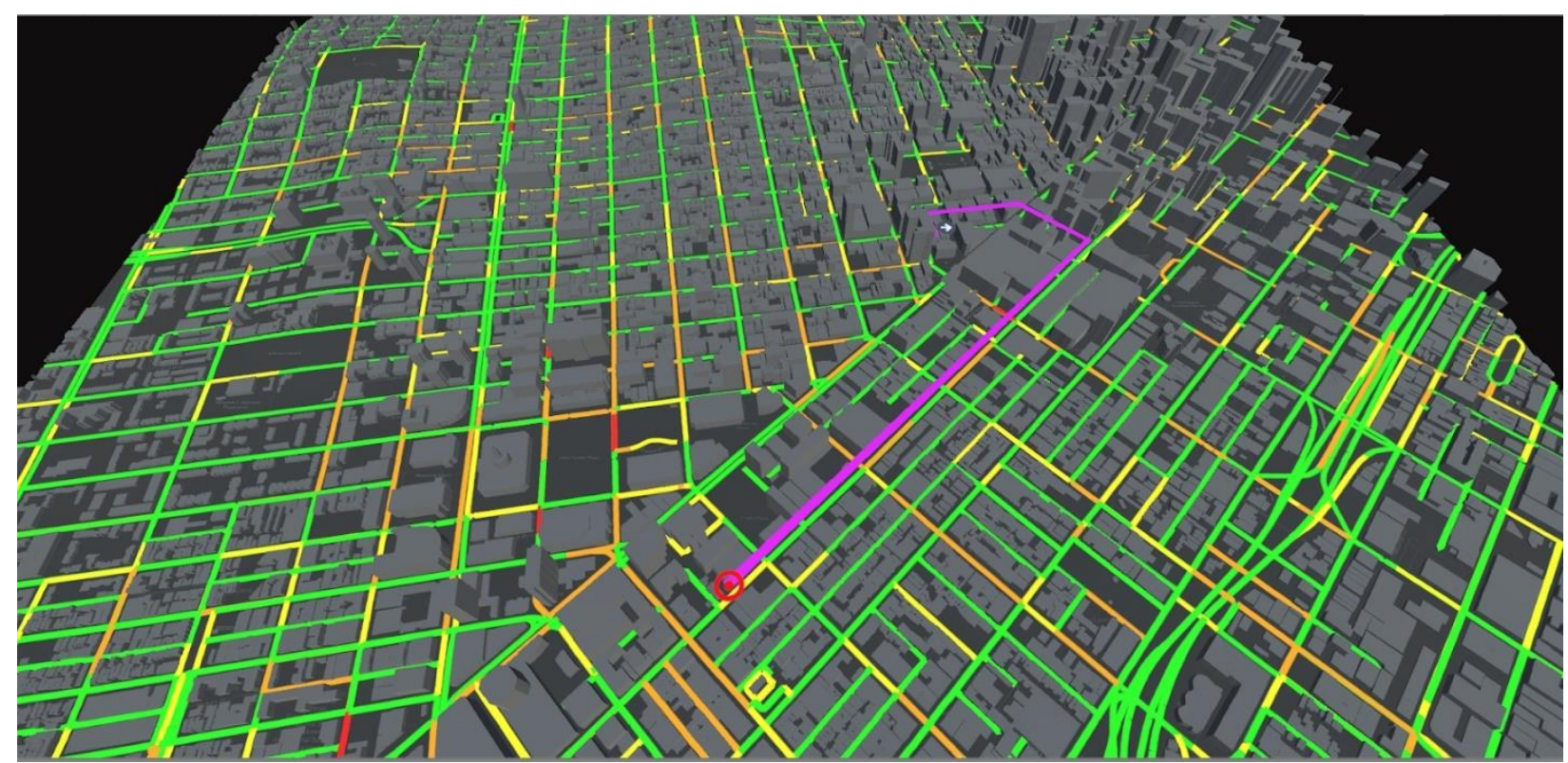

Figure 1. Visualization of people's average flow registered by GPS.

Some researchers have introduced parallel architectures such as graphics processing unit (GPU). These works take into consideration account visibility and different level of detail. This way faster rendering is achieved. An adaptive level of detail is used as well and a dynamic vertex buffer and index buffer that allows geometry to be generated at any point during grammar derivation on the GPU. It is important to address that this simulation must run at interactive frame rates (at least 30 frames per second). Thalmann and Boatright [Thalmanno9, Boatright2o13] stated that additional challenges such as Variety in both appearance and animation and behaviors.

Steering Behavior

Pedestrian steering behaviors or pedestrian motion involves the behavior of an individual by considering the other members of the crowd. According to Pettre [Pettre2009], steering has a big influence to get a plausible and a realistic crowd, and that is the main motivation behind being included as a part of the simulation workflow.

To address steering behavior, researchers have proposed different approaches following two alternatives. The first one is to deal the crowd as macroscopic phenomena. In this case, the crowd is simulated by using PDEs (Partial Differential Equations) with physic models treating the crowd as a whole [Shinohara2o11].

However, physics models have problems in simulating complex behaviors, since a fluid particle does not have a preferred direction of motion, does not have goals and cannot make decisions [Dai2013]. An alternative to the macroscopic approach is to deal with every agent in the crowd individually. This approach is called microscopic. Among the microscopic algorithms used to simulate dense crowds we have three main categories: vector based, agent based and data-driven techniques. One of the first research centered on microscopic simulation of autonomous virtual agents and their steering behaviors was presented by [Reynolds1987]. Several years later Helbing et al. presented the social forces model, where each agent tends to move at a desired velocity while applying friction and repulsion forces against obstacles and other agents [Helbing1995]. According to the authors, social forces are well suited for pedestrians in normal, or known, situations. The social forces model has been extended to support social groups of related pedestrians by Moussaïd et al. [Moussaid2010]. These kinds of methods can produce realistic results for specific situations or do not provide group steering behaviors. To do so, many finely tuned specific rules are required. As an example of the later, the work of [Lakoba2oo5] presented a modified version of Helbing's social force model proposed up to 24 parameters to set up. Sometimes tuning several parameters is a challenging task and often requires a lot of trial and error, this being an impediment 
to using this kind of methods for authoring tools. Particularly in the cases when it is necessary to add new behaviors and a new set of rules needs to be defined. Data-driven techniques generate behavior models by recording real pedestrian trajectories in video sequences. This approach generates several example situations and actions for the characters. Lerner et al. [Lerner2007] used video samples to construct a large example database. This database contains the data necessary to simulate individual trajectories. The amount of memory required to store the examples it ranges between $50 \mathrm{MB}$ to $15 \mathrm{O} \mathrm{MB}$. Containing up to $42 \mathrm{~K}$ of trajectories. On the other hand, Lee et al. [Lee2007] used the environment and the motion of nearby agents observed in video sequences to determine the moving trajectories of each simulated agent. Focusing its work on recreating groups dynamics rather than individual steering. In addition [Sun2o11] presented a data-driven collision avoidance algorithm and recently Charalambous et al. [Charalambous2014] presented a method for the synthesis of steering behaviors by abstracting the pedestrians steering behaviors in a structure called perception-action graph. Data-driven works usually don't model social group behavior and when they do the database grows significantly. In conclusion, data-driven models create very realistic results but require many examples and large amounts of memory to cover the complexity of social human behavior.

\section{Data Driven Crowd Visualization on Urban Areas}

\section{Architecture}

As mentioned before, the process of creating a complex urban environment is not a trivial task, many variables are involved in the process. Computational resources must be addressed when creating large scenes in consequence memory consumption becomes bigger for every additional element added to the virtual world. Nevertheless, memory is not the only problem, since these scenarios also consider high density crowds within, thus the time consumed by processing the simulation must be properly bounded to ensure an acceptable performance. Our goal in this proposed workflow is to ensure the simulation and the visualization of the virtual urban environment runs at interactive frame rate (at least 30 frames per second), to achieve this we divide this task in two different stages, the simulation stage and visualization stage, the completion of these two stages allows to the system to generate a plausible urban environment into the simulation.

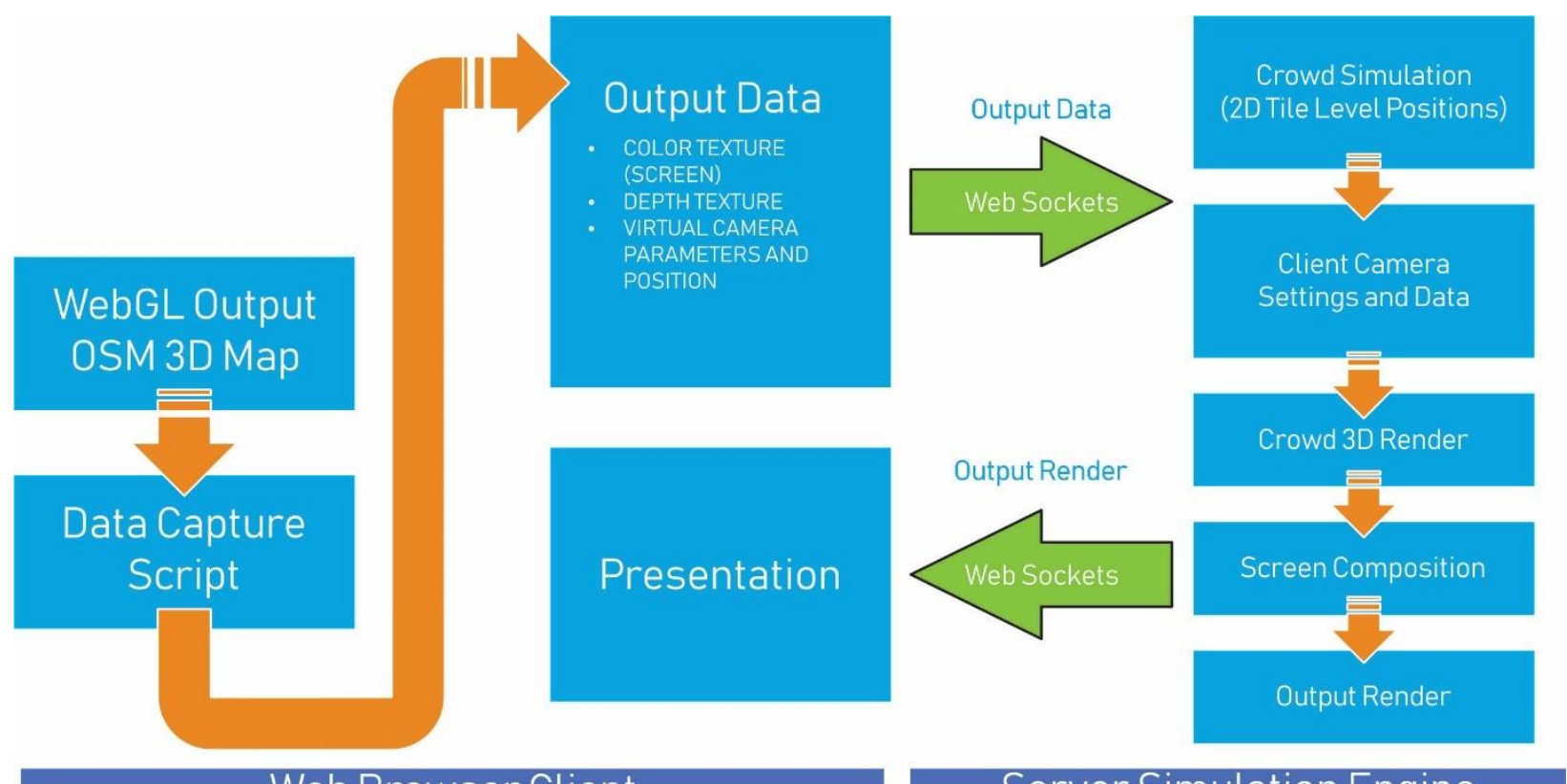

Web Browser Client

Server Simulation Engine

Figure 2. Visualization Engine Architecture. 
Figure 2 gives an overview on how the pipeline is currently working, we make our simulations using a webserver which allows to communicate within the application to fetch and process important data. The client initializes local map information to construct the environment, here we load all the requirements and begin the simulation, for instance, we fetch the OSM (Open Street Map) 3D map and the output is sent to the server where data is synchronized and get the final render. Figure 3 shows an example of the depth buffer also known as Zbuffer, this buffer is generated by rendering an extruded scene from the urban environment.
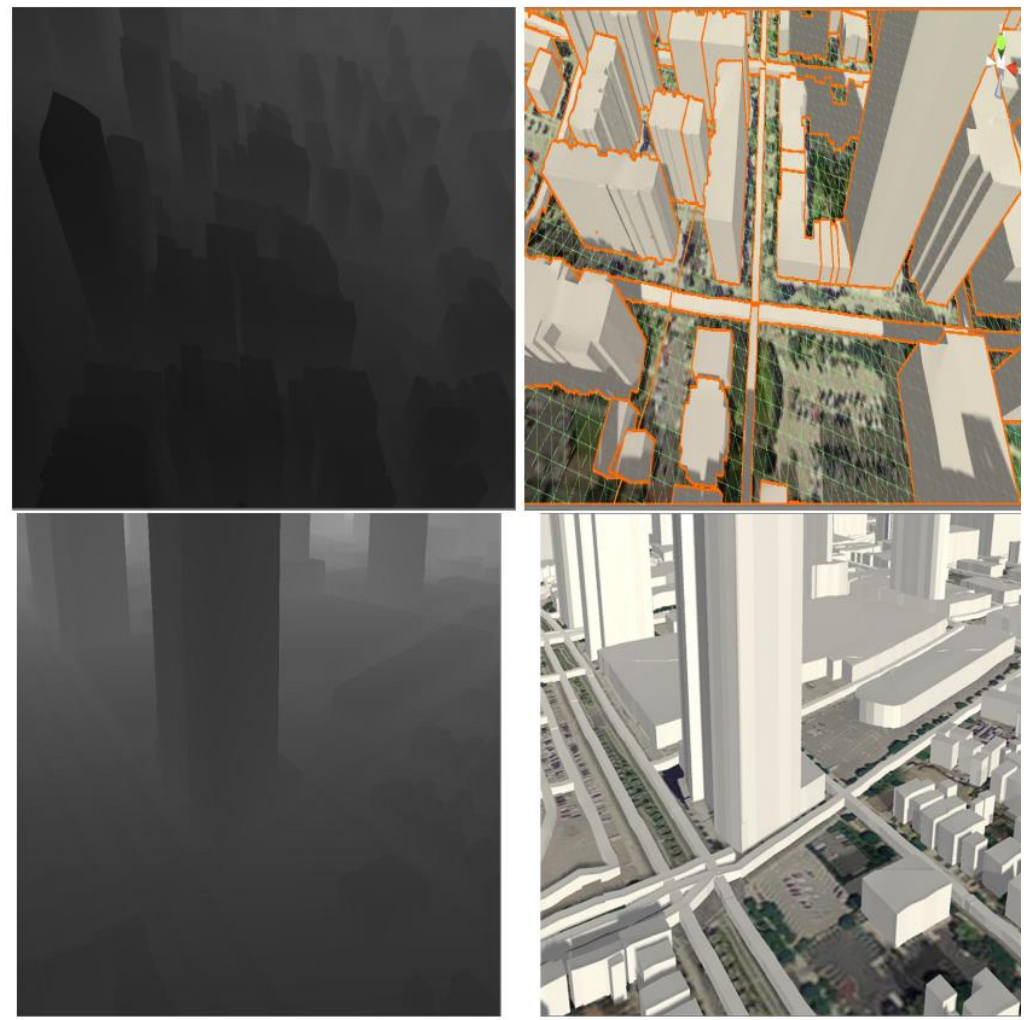

Figure 3. Z-buffer generated from OSM data.

Under this conception our client (a web browser) is used to visualize the urban crowd, therefore our server will be a dedicated process running the crowd simulation in an urban map scene. The server process uses web-sockets to establish a communication channel between the server and the browser, the general idea of communication between server and client is depicted in figure 2. On the following section we detail the jobs performed both by the client and the server.

\section{Client Pipeline}

In the client side we have coded a script in a web page that uses a lightly modified version of open Source Tangram, which is a visualizer of urban environments in $3 \mathrm{D}$, using Open Street Maps (OSM) geometry as input. The output is an interactive version of a city using real world information, which allows the users to navigate freely in the environment, our main focus in this stage is to synchronize the camera with the information that is displayed and merge it with our simulation, whenever the user applies any transformation to the camera, (i.e rotation or translation) we extract the depth buffer (DBF) of the scene, we also retrieve the scale in pixels per meter of the current zoom level, the $2 \mathrm{D}$ position relative to the current map, and the RGB of that specific frame. This is used to reconstruct the scene later and composite it with the crowd. 


\section{Server Pipeline}

The server side is the one that performs all the important data processing, all the process starts when the master process initializes the simulation and render parameters, the slave processes are also initialized, and will be running and simulating a predefined Map (in this case a part of an arbitrary city). The master process will spawn a server process with the web sockets protocol listening for a client. When the client performs a connection with the server, the initial simulation parameters are processed (world map position the most important). After that, the slave processes are taken from the idle state to a simulation state, the relative camera position and important data are loaded from the sent client frame buffer and the camera position is distributed from the master process to the slave process of the camera, along with other necessary render parameters. This scene is rendered from every slave process camera's point of view to a texture. The rendered scenes textures are sent to the master process, and the image composition is performed by the master process. Finally, the output render is sent to the client by the server process, all this will be repeated in an infinite loop until the client close the current connection or a prudent time without response from the client will be expired.

\section{Scene Compositing}

Compositing in OpenGL is a straightforward process and its implementation on the GPU shading language also. To create a compositing we only need a fragment shader that writes the depth and the output color of every screen pixel for each composited image in our pipeline; as an example if we have $\mathbf{n}$ processes rendering a part of a crowd in a separated Frame Buffer Object (FBO) to a texture along with its depth buffer, the master process will take each one of the output render textures and will render $\mathbf{n}$ times simply writing the depth value (usually glFragDepth ) and the texture output color using the GL DEPTH TEST option enabled to allow the render automatically discard the all the fragments, with exception of the closest one written by the master process, on figure 4 we have the Join of two computational jobs to obtain a composite image, the first component corresponds to the output of four partial rendering processes of the crowd characters and the second one corresponds to the process that generates the background.

Final Render
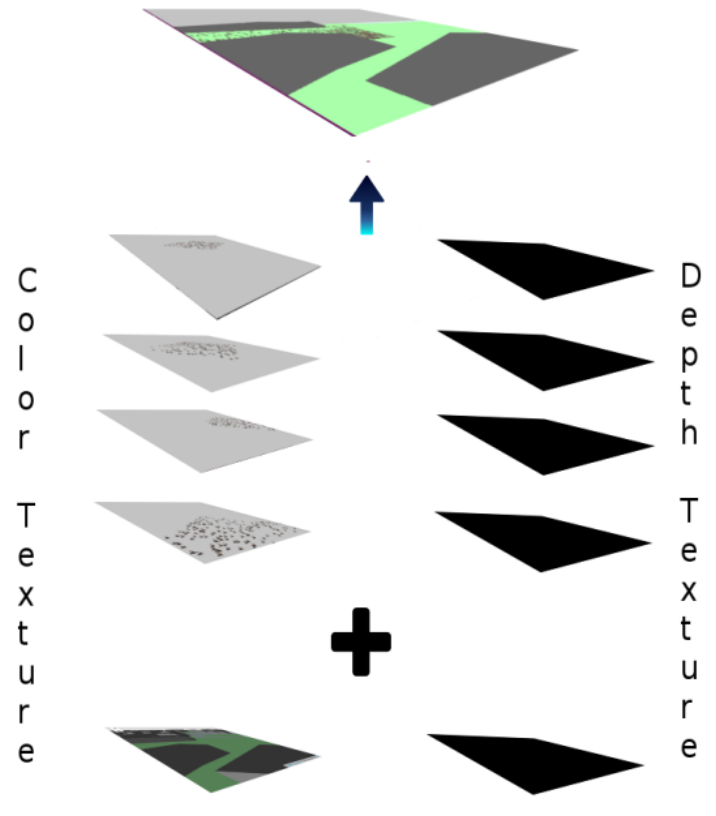

Background

Figure 4. Join of two computational jobs yielding a composite image. 


\section{Visualization of urban environment}

There are several techniques employed to create complex urban scenarios, some use a detailed model generated from scans and photographs, some others simply use extrusion to generate buildings of the appropriate height from the contours in the 2D map and height information. We chose to use "MapzenTangram", where as stated in its manual, we can generate extruded buildings by using a function that applies to polygons and lines, and returns such features drawn with the polygons draw style into $3 \mathrm{D}$ space along the $z-$ axis. this function raises elements drawn with the lines draw style straight up from the ground plane, using the values in the feature's height and $\min _{\text {height }}$ properties. Our web application will call Mapzen Tangram to generate an appropriate $3 \mathrm{D}$ view of the city, using extrusion, whenever the mouse is moved. from this view, we generate the color and depth buffers by rendering (as explained in the following). these buffers and the appropriate camera parameters are used to generate the rendering of the crowd and generate the final composite image.

Data Driven Steering Behavior

Finally, to make each pedestrian inside the virtual environment move realistically, we need a system able to simulate the subtle variations on the steering behavior of pedestrians without needing large amounts of data, we extracted from videos the steering movements of pedestrians and store them as examples to create a knowledge base of learned steering behaviors. To model social group phenomena, we used group social forces proposed by Moussaïd [Moussaid2o10]. In this manner, we get the best features of both models.

Knowledge Base Conformation

The first goal of the process is to obtain from the video a record of each path followed by each pedestrian. Each path is processed and later use to create a data structure for store these steering behaviors. We use a video shot looking directly down on the subject, also called bird's eye view shot. This view is chosen to avoid occlusions and artifacts in the tracked paths usually caused by inconsistent mappings between the real world and the image coordinates. A general plane to plane projective mapping is given by the equation:

$$
x=\frac{a u+b v+c}{g u+h v+i} ; y=\frac{d u+e v+f}{g u+h v+i}
$$

Eq. 1

From the above $(u, v)$ represents world coordinates, $(x, y)$ correspond to images coordinates, and " $a, b, c, d, e, f, g, h, i$ " are calibration constants. Using a top-down shot the equation 1 tends to be closer to:

$$
x=a * u ; y=e * v
$$

Eq. 2

The scene is split into background and foreground using the codebook algorithm [Schreiber2009]. Foreground regions are considered region of interest "ROI". These ROIs are passed one by one to an LBP feature extraction module and then are classified as human head or not. Once a human head is detected, a point is associated with it. Each detected point is tracked over every frame using the pyramidal version of the Lucas-Kanade tracker implemented on the OpenCV library (see figure 5), The pipeline of human tracking starts with top view shots. then the human heads are segmented and tracked to get the path of every pedestrian in the scene. 

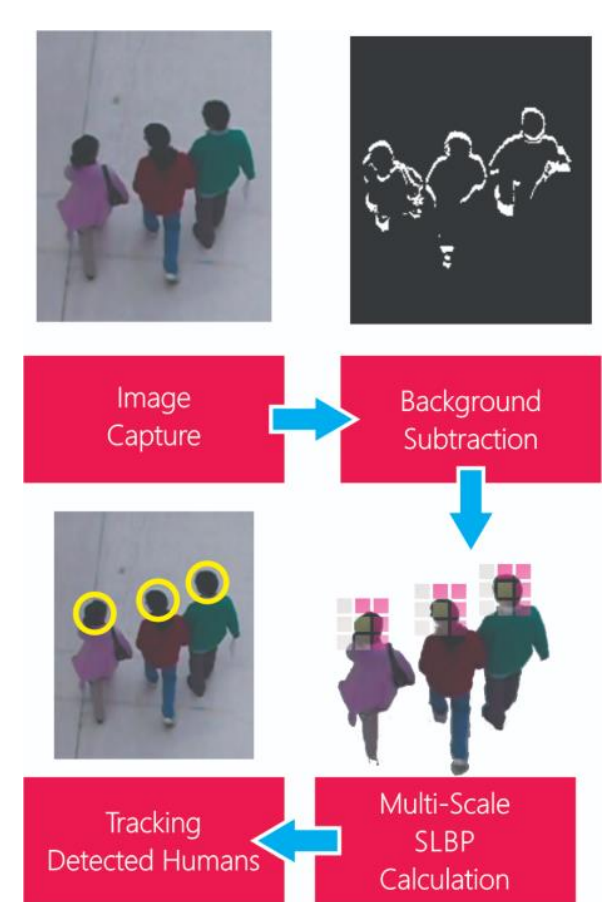

Figure 5. Human tracking pipeline.

A trajectory of each pedestrian $\tau_{k}$ is defined as a set of $N$ displacements $\delta_{i}$ from position $P_{i}\left(x_{i}, y_{i}\right)$ to $P_{i+1}\left(x_{i+1}, y_{i+1}\right)$. In consequence each displacement $\delta_{i}$ is given by:

$$
\delta_{i}=\left(x_{i+1}-x_{i}, y_{i+1}-y_{i}\right)
$$

Therefore $\tau_{k}$ is conformed as:

$$
\tau_{k}=\left\{\delta_{0}, \delta_{1}, \ldots, \delta_{N}\right\}
$$

Once all the trajectories have been collected, the next step is to extract a tuple of set of features and actions to build the behavior cores [Lee2007]. We propose a set of 3 features which have strong influence in the steering decision of a pedestrian: normalized pedestrian velocity, closeness to goal and obstacle code.

Before explaining how to get the normalized pedestrian velocity, we need to describe how to get the goal vector, which is expressed in the following equation:

$$
\text { goal }=\sum_{i=0}^{N} \delta_{i}
$$

The data we have collected is made of a wide range of starting points and destinations of each pedestrians. To better deal with each agent's decision-making process, is necessary normalizing the vectors that describe movement. Given the fact that the goal vector is defined as the sum of the partial displacement of a pedestrian, we decided to transform the global coordinate system obtained in the tracking visual phase to a local one, offsetting all displacements to make the goal vector pointing toward the local "y" axis. Therefore, given a goal vector, we use a vector $\widehat{e_{2}}=(0,1)$ to get a normalization angle, which is the angle needed to align the goal vector with "y" axis. We call it a normalization angle which is calculated using the following equation:

$$
\eta=\cos ^{-1}\left(\frac{\hat{e}_{2} \times \text { goal }}{\left|\hat{e}_{2}\right| \times \mid \text { goal } \mid}\right) \text { eq. } 6
$$


Given a vector displacement $\delta$. The normalized version $\gamma$ of that vector according to angle $\eta$ is given by the following expression:

$$
\vec{\gamma}=\left|\begin{array}{l}
\vec{\delta}_{x} \times \operatorname{Cos}(\eta)-\vec{\delta}_{y} \times \operatorname{Sin}(\eta) \\
\vec{\delta}_{y} \times \operatorname{Cos}(\eta)-\vec{\delta}_{x} \times \operatorname{Sin}(\eta)
\end{array}\right|
$$

Therefore, once we calculate the normalized angle, the goal vector is rotated or normalized using equation 6 . Producing the goalN, notice the new vector has no "x" component due to normalization. See figure 6.

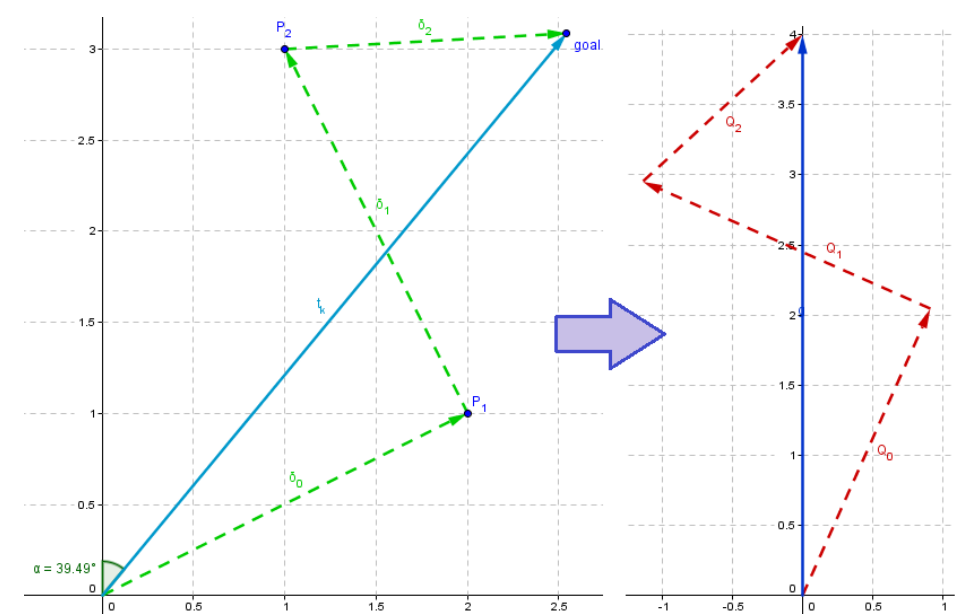

Figure 6. This figure shows on the left a path obtained from the tracking process, on the right the normalized version.

The second feature is the velocity, this factor comprises the rate of change of time, $\Delta t$ of the displacement of the pedestrian as a function of time. The velocity given by equation 7 provides part of the component of behaviors that describe collision avoidance.

$$
\vec{v}_{i}=\frac{\gamma_{i+1}-\gamma_{i}}{\Delta t}
$$

Once the velocity has been calculated it is reoriented by the normalization angle $\eta$. From here we will call as $\phi_{i}$ to all the velocity vectors normalized using equation 6.

The next feature is the closeness to goal, this feature outlines how close (in percentage) the pedestrian is from its current position to the destination observed in the trajectory dataset. The closeness to goal factor is defined by:

$$
\sigma_{i}=\frac{\overrightarrow{r_{r}} \cdot \text { goal }_{i}}{\text { goal }_{\text {ix }}^{2}+\text { goal }_{i y}^{2}}
$$

This feature is important in our model given the fact that it affects directly the steering behavior of a pedestrian making able to a virtual pedestrian to react differently when is close to its goal.

The last feature that makes up our state vector is the obstacle code.

The obstacle code $\varphi$ is a factor that is calculated by using eight discrete radial regions. This kind of subdivision has been frequently used to capture the influence of the neighborhood in data-driven approaches [Torrens2o11]. Perceptual studies have demonstrated that regions toward the intended direction have a larger radius of influence on the trajectory of pedestrians [Lerner2007] that fact lead us to introduce a slight difference consisting on incrementing the radius of the section pointing toward the direction of pedestrian's motion (see figure 7). The angle of obstruction $\beta$ of a pedestrian $j$ in the neighborhood of a pedestrian $i$ walking at a velocity $\boldsymbol{v}_{\boldsymbol{i}}$ is given by: 


$$
\begin{gathered}
\alpha=\operatorname{atan} 2\left(\vec{e}_{i, j x}, \vec{e}_{i, j y}\right)-\operatorname{atan} 2\left(\vec{v}_{i y}, \vec{v}_{i x}\right) \\
\alpha_{1}=\left\{\begin{array}{cc}
\alpha+2 \cdot \pi & \alpha<0 \\
\alpha & \alpha \geq 0
\end{array}\right.
\end{gathered}
$$

Eq. 10

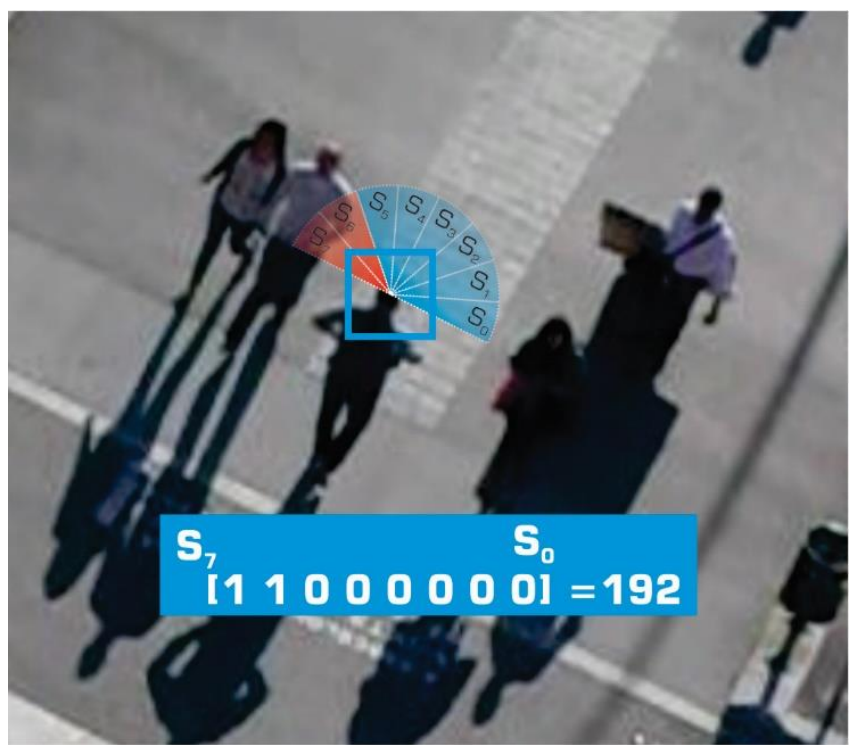

Figure 7. The space around the agent establish the obstacle code $\varphi$.

From the equation 9, the $e_{i j}$ vector is pointing from pedestrian $i$ to $j$. With the angle of obstruction $\alpha_{1}$ the next quadrant adjustment is performed:

$$
\beta=\left\{\begin{array}{cc}
\alpha_{1}+\frac{\pi}{2} & \alpha_{1}<\frac{\pi}{2} \\
-1 & \frac{\pi}{2} \leq \alpha_{1}<\frac{3 \pi}{2} \\
\alpha_{1}-\frac{3 \pi}{2} & \alpha_{1} \geq \frac{3 \pi}{2}
\end{array}\right.
$$

Finally, the quadrant obstructed by pedestrian $j$ is:

$$
\varphi=\left\lceil\frac{8 \times \beta}{\pi}\right\rceil
$$

The set of features $\phi_{x}, \phi_{y}, \sigma, \varphi, A_{x}, A_{y}$ define a state vector $S_{i}$ (see Eq. 13). In this case $A_{x}, A_{y}$ forms a 2D vector which represents the motion performed by the pedestrian, also known as a pedestrian's state vector. All the vectors $S_{i}$ which match the same goal goal $k_{k}$ are packed in a look-up table $\Lambda_{m}$ see Eq. 14 .

$$
\begin{array}{r}
\overrightarrow{S_{\iota}}=\left[\Phi_{i x}, \Phi_{i y}, \sigma, \varphi, A_{x}, A_{y}\right] \\
\Lambda_{m}=\left[\vec{S}_{0}, \vec{S}_{1}, \ldots, \vec{S}_{N}\right]
\end{array}
$$

Therefore table $\Lambda_{m}$ represents our knowledge-base. The input for the knowledge base will be a state $\mathbf{S}$, the system finds the closest match between the incoming state vector inside the knowledge-base. Once we have a match, the system returns the action vector $\left(A_{x}, A_{y}\right)$.

Social Data Driven Simulation Model

Finally, the resulting steering vector of a pedestrian is modeled according to equation 15 . The A component of the steering force is given by the knowledge base as a function of the pedes- 
trian state presented in the simulation. The rest of the components are given by $\vec{f}_{i}^{\text {group }}$ which is the last component of the Moussaïd model of group social forces [Moussaid2o1o]. This fact allowed us to avoid demanding more memory resources to store persistent data related to group formations in the knowledge base. We chose the group force equation presented by Moussaïd because reproduces faithfully the group formations in pedestrians.

$$
\frac{d \overrightarrow{v_{l}}}{d t}=\vec{A}+\vec{f}_{i}^{\text {wall }}+\sum_{j \neq i} f_{i, j}+\vec{f}_{i}^{\text {group }}
$$

\section{Results}

\section{Crowd Visualization}

For our experiments we execute the simulation and visualization process in a workstation with these characteristics: Intel Core i7-4820zK CPU @ 3.70GHz 8, 16Gb of RAM, GeForce GTX TITAN Black, 2880 CUDA Cores and 6Gb of RAM.

Since our simulation engine was developed previously, it is ready to be executed in a cluster environment. However, for this paper, and to have a better control of the environment, avoiding inter-node communication problems, we focus in the integration of the urban scenarios, and present results obtained by running both client and server in the same workstation. We execute the simulation with five, ten and seventeen "mpi" processes. One of them manage the urban environment and do the composition, the rest are used to simulate and render the agents. All

the client-side interaction and scripts where developed and tested in Google Chrome for Linux.

The maximum number of agents in the simulation is restricted to the GPU's memory, in this workstation the limit was 80000 agents.

Each frame the simulation engine updates the agents positions to be subsequently rendered as full characters way by the visualization engine, as shown in Figure 8 and 9. We still have some work to do in improving the quality of the renders in the client side without making the latency increase. Our tests have been tried with a better-quality render in the client's side, but the latency generated by the texture capture cycles, affects negatively the client's user experience and generates a set of artifacts in the client side because of large render cycles.

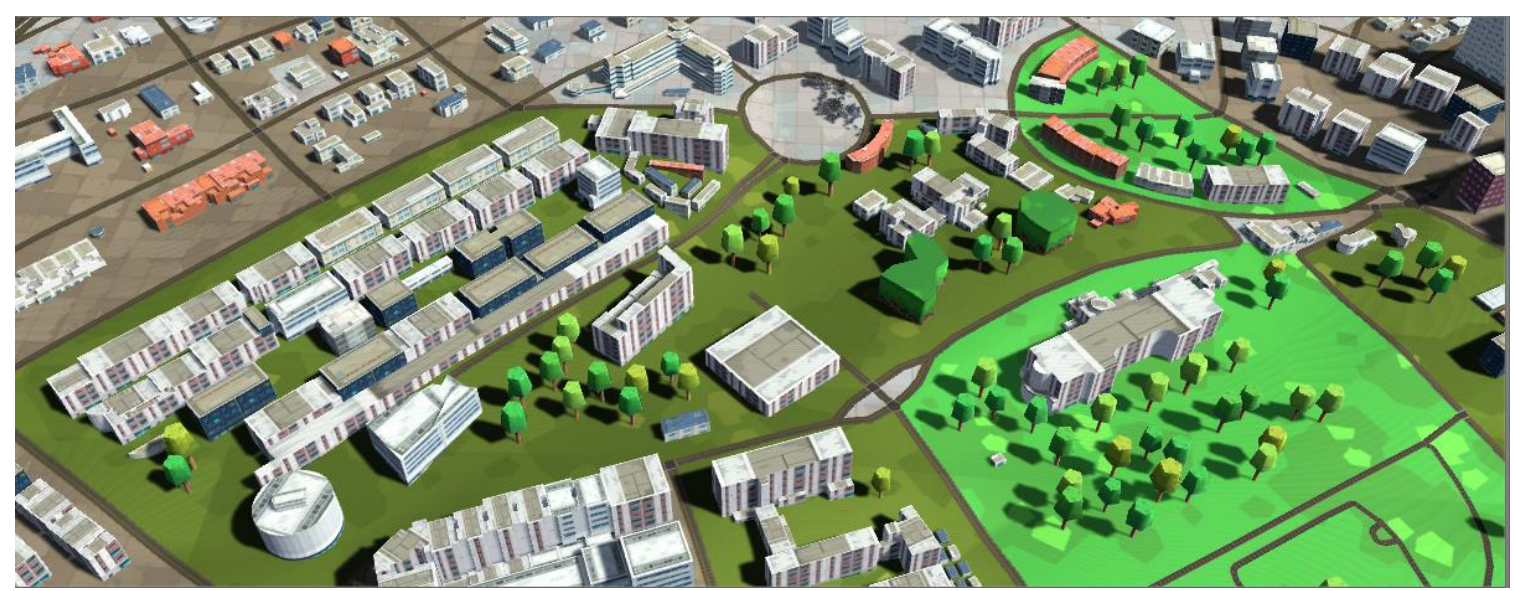

Figure 8. Visualization of the city from an aerial view. 


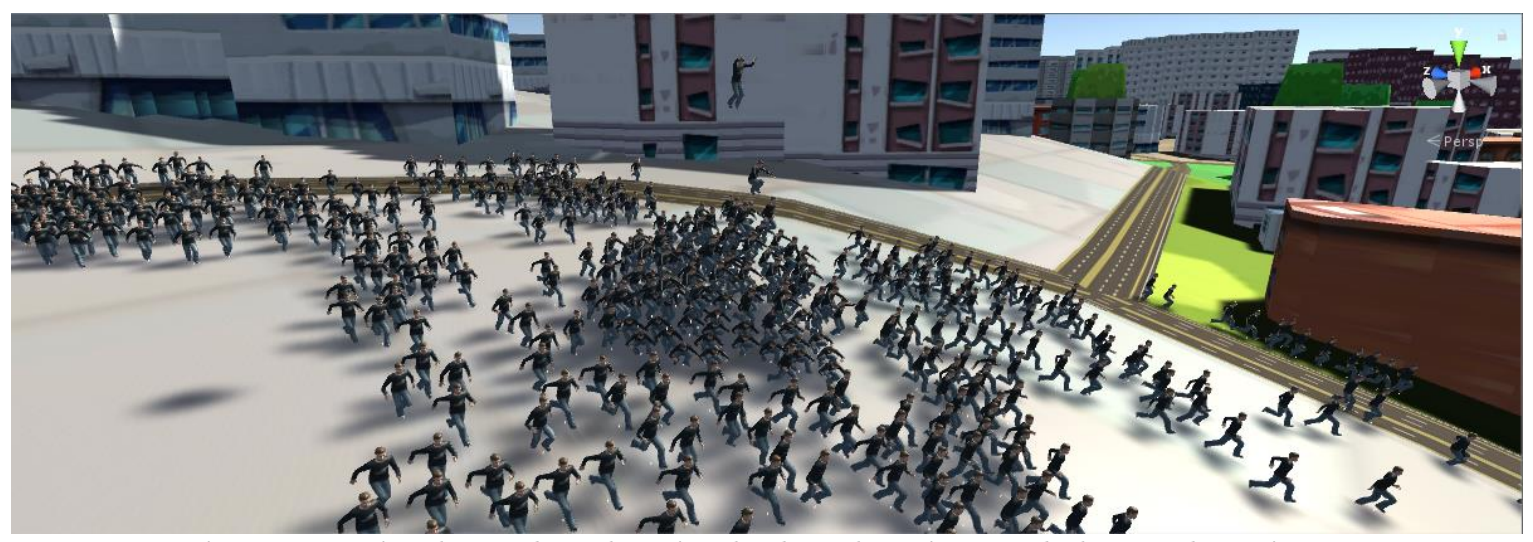

Figure 9. Final render that includes the city and the pedestrians.

\section{Steering Behavior}

Aiming to evaluate the simulation of the pedestrian steering motions, we employ a metric based on entropy as a measure of the size of the predicted error.

In the scientific community, one can find several proposals for evaluating the quality of a crowd simulation. Some of the existing work focuses on evaluating crowds based on quantifying the extent to which people respond realistically to virtual events and situations [Pelechano2008]. A similar perception-like proposal is presented by Ahn et al. [Ahn2012] who conducted a test user study in a four-screen CAVE to compare different crowd simulations. Other proposals such as Kapadia [Kapadia2o11] use a set of metrics based on path smoothness, collisions between virtual characters and path lengths.

Previous approaches were designed to compare the results in synthetic environments but are not suitable to be used to compare the similarity between a simulation and the real behavior of a pedestrian by the other hand the entropy has proven to be applicable to data generated with small and large number of pedestrians in sparse and dense scenes [Guy2012].

The measure of entropy is defined as follows: Given a state of a real scene $Z_{k}$ the difference between the action vector $A\left(Z_{k}\right)$ and the next state $Z_{k+1}$ is calculated giving an entropy vector. In this case the total entropy for a given path is calculated using equation 16 . In this case, the smaller the entropy the better the simulation.

$$
E n=\sum_{k=0}^{N}\left\|Z_{k+1}-A\left(Z_{k}\right)\right\|
$$

Eq. 16

We ran a test measuring the entropy for a single path followed by a pedestrian again different simulation models: vector based, data-driven based and our hybrid model (Fuzzy data-driven with group forces "FDDGF"), we called this test "Single Agent Entropy" the result of this test is presented in figure 10 where the sample size was of fifty random pedestrians walking alone.

\section{SINGLE AGENT ENTROPY TEST}

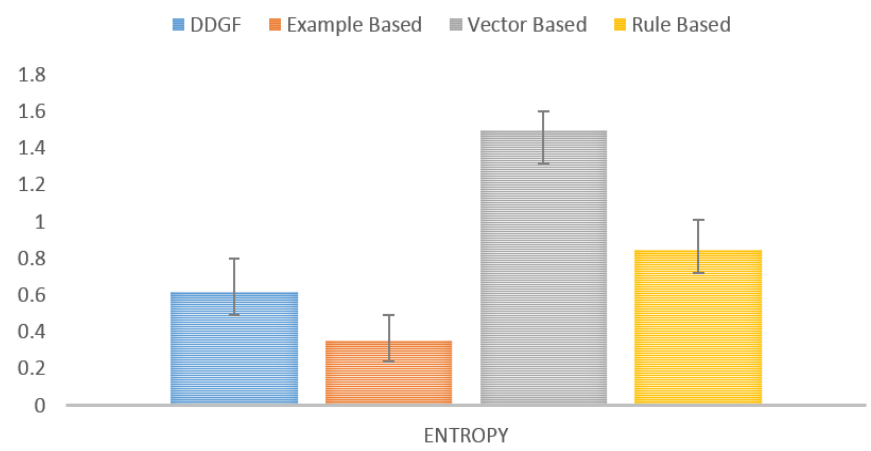

Figure 10. Single Agent Entropy Test with 50 pedestrians. 
Our system ranked in the second place (The lower the better) just slightly above over pure data driven techniques (example based). It is a matter of fact that Data-driven methods closely reflect the behavior of pedestrians in real scenes, but a major drawback in this approach is that they require large amounts of data and scaling those systems sometimes becomes unfeasible. On the other hand, vector-based methods and rule-based methods demand less memory but they need hard fine-tuning parameters that govern agent behaviors, which can be a very demanding task.

The second test we ran was quite similar to the first one but instead of having one pedestrian, the scene was composed of many individual pedestrians not belonging to any group. These results can be seen in Figure 11.

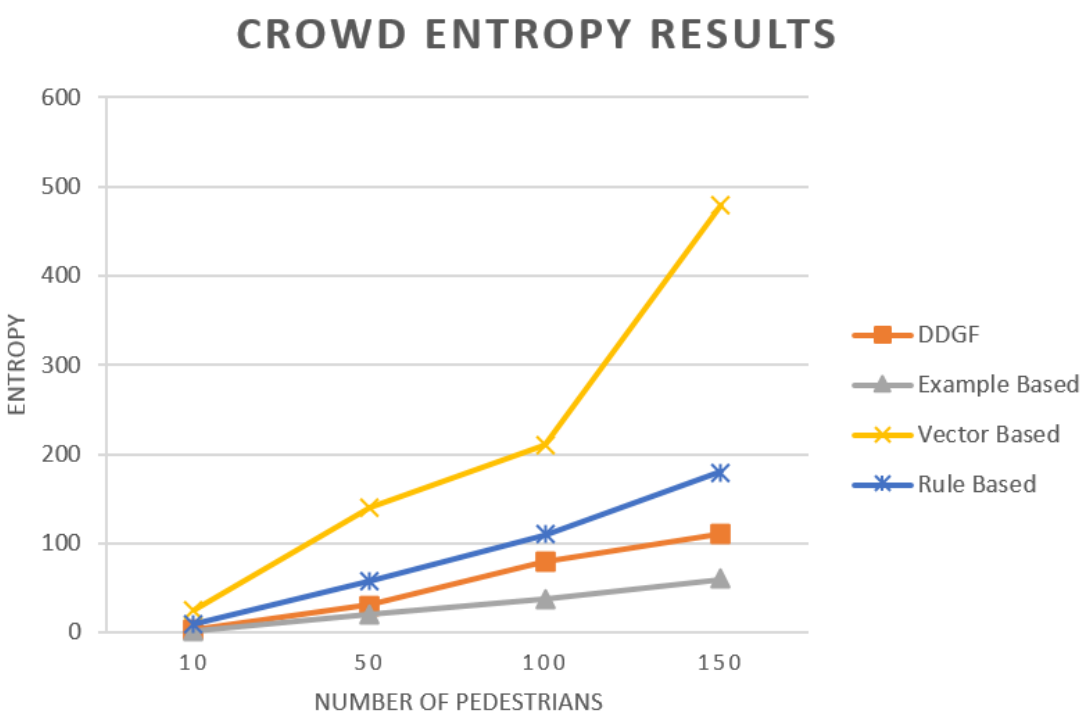

Figure 11. Crowd Entropy Test.

In this simulation of crowds without social group formations again the data-driven method achieves the best result meanwhile our method reaches the second place on the low entropy measures just slightly below the example-based method which consumes more data.

The last test done was in scenes where social groups up to 4 people are presented. We called this test "Crowds with social groups" (Figure 12), on this test our method achieves the best result getting close to data-driven techniques, but also the data-driven presents the best results.

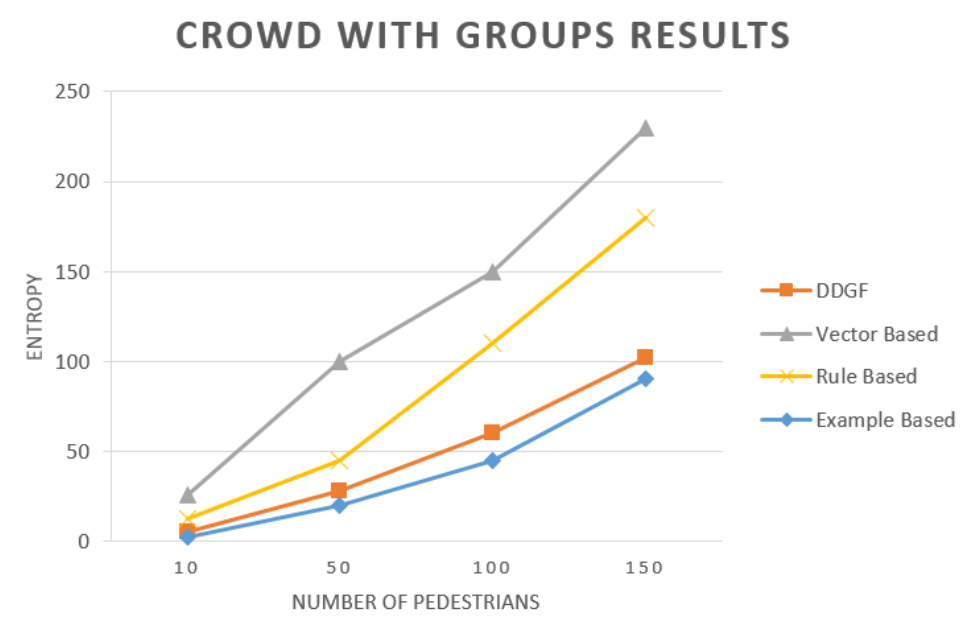

Figure 12. Crowd with Social Groups. 


\section{Conclusions}

Our experiments show that mixing data-driven methods with group forces allow us to achieve results comparable to those obtained with data-driven systems but using less memory and avoiding fine tuning parameters jobs. Figure 13 shows the comparison of our method again the above mentioned in terms of number of parameters and memory that make our system usable even in devices with limited memory and computational power.

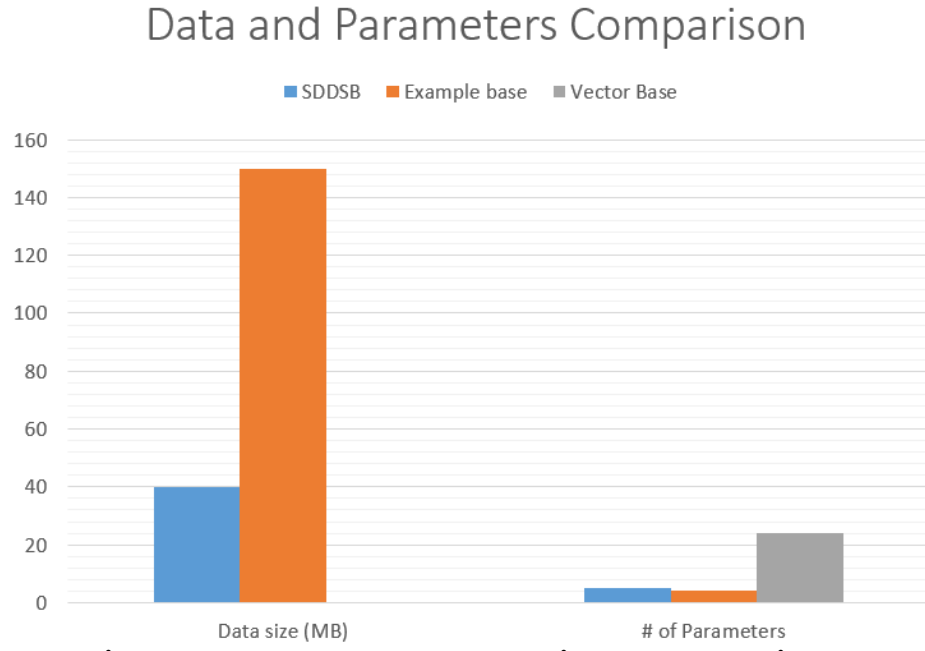

Figure 13. Data Consumption Comparison.

The simulation generated by our method produces a steering behavior learned from real videos, resulting in similar trajectories to those observed in real pedestrian steering. Our DataDriven technique allow us to get plausible results since the system captures some of natural movements presented in real scenes

Crowd simulation is a field that is constantly challenging and testing graphics hardware since most of the times the available resources are not enough to simulate a crowd without any optimization technique, such as LOD or compositing. This field demands a lot of computational power and resources, so an efficient way to assign memory and processing power is mandatory to achieve the desired results. Applications for virtual city generation range from research and educational purposes such as urban planning and creation of virtual environments for simulation. Movie and game industries have a high demand for quick creation of complex environments in their applications, since they are in constant need for more art assets that form virtual worlds to support interaction, training, evaluation, virtual sets, and other uses. Security, crisis management and virtual training can take advantage of this environments as well. In the present work we described a workflow able to visualize and simulate 80000 agents per frame inside an urban environment, we incorporate steering behavior to each pedestrian, and GPS information to manage the flow of people inside the urban environment. The future steps will consist in add realism to the buildings rendering.

\section{Acknowledgements}

Special thanks to SECTEI in Mexico for providing part of the founding for the present research, also we must express our gratitude for all the team that conforms the Barcelona Super Computing Center for facilitate the equipment and the infrastructure for the project.

\section{References}

[Ahn2012] AHN, J; WANG, N; THALMANN, D; ANDBOULIC, R. 2012. Within Crowd Immersive Evaluation of Collision Avoidance Behaviors. Vrcai, 231-238. 
[Boatright2o13] Boatright, C.D; Kapadia, M; Shapira, J.M; Badler, N.I.: Context-sensitive data driven crowd simulation. Proceedings of the 12th ACM SIGGRAPH International Conference on Virtual-Reality Continuum and Its Applications in Industry - VRCAI '13 pp. 51-56 (2013), http://dl.acm.org/citation.cfm?doid=2534329.2534332

[Charalambous2014] CHARALAMBOUS, P; AND CHRYSANTHOU, Y. 2014. The PAG Crowd: A Graph Based Approach for Efficient Data Driven Crowd Simulation. Computer Graphics Forum 33, 0, 95-108.

[Dai2013] DAI, J., LI, X; ANDLIU, L. 2013. Simulation of pedestrian counter flow through bottlenecks by using an agent-based model. Physical A: Statistical Mechanics and its Applications 392, 9(May), 2202-2211.

[Essen2008] Van Essen, R.: Maps Get Real: Digital Maps evolving from mathematical line graphs to virtual reality models, pp. 3-18. Springer Berlin Heidelberg, Berlin, Heidelberg (2008)

[Guy2012] GUY, S. J; VAN DENBERG, J; LIU, W; LAU, R; LIN, M. C; AND MANOCHA, D. 2012. A statistical similarity measure for aggregate crowd dynamics. ACM Transactions on Graphics 31,6, 1.

[Helbing1995] HELBING, D; ANDMOLNÁR, P. 1995. Social force model for pedestrian dynamics. Physical Review E 51, 5 (May), 4282-4286.

[Kapadia2o11] KAPADIA, M; WANG, M; SINGH, S; REINMAN, G; ANDFALOUTSOS, P. 2011. Scenario Space: Characterizing Coverage, Quality, and Failure of Steering Algorithms. ACMSIGGRAPH / Eurographics Symposium on Computer Animation (SCA) 1, 53-62

[Lakoba2005] LAKOBA, T. I. 2005. Modifications of the Helbing-Molnar-Farkas-Vicsek Social Force Model for Pedestrian Evolution Simulation 81, 5 (May), 339-352.

[Lee2007] LEE, K; CHOI, M; HONG, Q; ANDLEE, J. 2007. Group behavior from video: a data-driven approach to crowd simulation. In Proceedings of the 2007 ACM, 109-118.

[Lerner2007] LERNER, A., CHRYSANTHOU, Y, AND LISCHINSKI, D. 2007.Crowds by Example. Computer Graphics Forum 26, 655-664.

[Loviscach2o06] Loviscach, J.: Deformations: Wrinkling coarse meshes on the GPU 25(3), 467-476 (Sep 2006)

[Moussaid2o10] MOUSSAÏD, M., PEROZO, N; GARNIER, S; HELBING, D; AND THERAULAZ, G. 2010. The walking behaviour of pedestrian social groups and its impact on crowd dynamics.PloS one 5, 4(Jan.), e10047.

[Navarro2o11] Navarro L., Flacher F., Corruble V.: Dynamic level of detail for large scale agent-based urban simulations. In The 1oth International Conference on Autonomous Agents and Multiagent Systems - Volume 2 (Richland, SC, 2011), AAMAS '11, International Foundation for Autonomous Agents and Multiagent Systems, pp. 701-708. URL: http://odl.acm.org.millenium.itesm.mx/citation.cfm?id=2031678.2031717.

[Pelechano20o8] PELECHANO, N., ANDSTOCKER, C. 2008. Being a part of the crowd: towards validating VR crowds using presence. Proceedings of the 7th international joint conference on Autonomous agents and multiagent systems, Aamas, 12-16.

[Pettre2009] PETTRÉ, J; ONDREJ, J; OLIVIER, A.-H; CRETUAL, A; ANDDONIKIAN, S. 2009. Experiment-based modeling, simulation and validation of interactions between virtual walkers. Proceedings of the 2009 ACM SIGGRAPH / Eurographics Symposium on Computer Animation 2009, 189.

[Reynolds1987] REYNOLDS, C. W. 1987. Flocks, herds and schools: A distributed behavioral model.ACM SIGGRAPH Computer Graphics 21, 4(Aug.), 25-34.

[Schreiber2009] SCHREIBER, D; ANDRAUTER, M.2009.GPU-based non-parametric background subtraction for a practical surveillance system.2009 IEEE 12th International Conference on Computer Vision Workshops, ICCV Workshops (Sept.), 870-877.

[Shinohara2o11] SHINOHARA, K.; AND GEORGESCU, S. 2011. Modelling Adopter Behavior Based on the Navier Stokes Equation. ISRN Mathematical Analysis 2011, 1-10.

[Silverira2006] Da Silveira, L.G., Musse, S.R.: Real-time generation of populated virtual cities. 
In: Proceedings of the ACM Symposium on Virtual Reality Software and Technology.

pp. 155-164. VRST 'o6, ACM, New York, NY, USA (2006), http://doi.acm.org/10.1145/1180495.1180527.

[Sullivan2002] O'Sullivan C., Cassell J., Vilhjalmsson H., Dingliana J., Dobbyn S., McNamee B., Peters C., Giang T.: Levels of detail for crowds and groups. Computer Graphics Forum 21, 4 (2002), 733\{741. URL: http://dx.doi.org/10.1111/1467-8659.00631, doi: 10.1111/1467-8659.00631.

[Sun2011] SUN, L; ANDQIN, W. 2011. A Data-Driven Approach for Simulating Pedestrian Collision Avoidance in Crossroads.2011 Workshop on Digital Media and Digital Content Management (May),83-85.

[Thalmann2oo9] Thalmann, D; Grillon, H; Maim, J; Yersin, B.: Challenges in Crowd Simulation. 2009 International Conference on Cyber Worlds pp. 1-12 (2009), http://ieeexplore.ieee.org/lpdocs/epico3/wrapper.htm?arnumber $=5279720$

[Thomsen2oo8] Thomsen, A., Breunig, M., Butwilowski, E., Broscheit, B.: Modelling and Managing Topology in 3D Geoinformation Systems, pp. 229\{246. Springer Berlin Heidelberg, Berlin, Heidelberg (2008)

[Torrens2o11] TORRENS, P; LI, X; AND GRIFFIN, W.A. 2011. Building Agent-Based Walking Models by Machine-Learning on Diverse Databases of Space-Time Trajectory Samples.Transactions inGIS 15, 67-94. 